\title{
Intracule functional models. IV. Basis set effects
}

\author{
Jason K. Pearson, ${ }^{\text {a) }}$ Deborah L. Crittenden, and Peter M. W. Gill ${ }^{\text {b) }}$ \\ Research School of Chemistry, Australian National University, ACT 0200, Australia
}

(Received 19 January 2009; accepted 30 March 2009; published online 24 April 2009)

\begin{abstract}
We have calculated position and dot intracules for a series of atomic and molecular systems, starting from an unrestricted Hartree-Fock wave function, expanded using the STO-3G, 6-31G, 6-311G, $6-311++\mathrm{G}, 6-311++\mathrm{G}(d, p), 6-311++\mathrm{G}(3 d, 3 p)$, and $6-311++\mathrm{G}(3 d f, 3 p d)$ basis sets as well as the nonpolarized part of Dunning's cc-pV5Z basis. We find that the basis set effects on the intracules are small and that correlation energies from the dot intracule ansatz are remarkably insensitive to the basis set quality. Mean absolute errors in correlation energies across the G1 data set agree to within $2 \mathrm{~m} E_{h}$ for all basis sets tested. (C) 2009 American Institute of Physics. [DOI: 10.1063/1.3122422]
\end{abstract}

\section{INTRODUCTION}

In the context of quantum chemistry, intracules are twoelectron distribution functions that contain information about quantities such as the distance $u=\left|\boldsymbol{r}_{1}-\boldsymbol{r}_{2}\right|$ between two electrons or their relative momentum $v=\left|\boldsymbol{p}_{1}-\boldsymbol{p}_{2}\right|$. The position intracule $P(u)$, for example, gives the probability of finding two electrons separated by a relative distance $u .{ }^{1-4}$ Likewise, the momentum intracule, $M(v)$, gives the probability of finding two electrons moving with relative momentum $v^{5,6}$

As functions that contain explicit two-electron information, it is natural to ask whether $P(u)$ and/or $M(v)$ provide a suitable foundation for the construction of post-HartreeFock (HF) correlation energy models. Unfortunately, it appears that neither is ideal for the prediction of the electron correlation energy, $E_{c}$, as illustrated by the isoelectronic series of heliumlike ions ${ }^{7}$ where, as the nuclear charge $Z$ increases, one finds that $\langle u\rangle$ decreases as $O\left(Z^{-1}\right),\langle v\rangle$ increases as $O(Z)$, and $E_{c}$ approaches a constant. This suggests that the product variable $s=u v$ may be the smallest basic unit required to model correlation energies and implies that an intracule-based correlation model containing information about both $u$ and $v$ is required for accurate correlation energies.

The Uncertainty Principle precludes the construction of a joint probability density in position and momentum space but we have managed nonetheless to generate phase-space intracules from the second-order reduced Wigner distribution, ${ }^{8,9}$ a joint quasiprobability for the two-electron position-momentum density. In this way, we have constructed the Omega intracule $\Omega(u, v, \omega),{ }^{10,11}$ which can be interpreted as the joint quasiprobability density for $u, v$, and $\omega$, the latter being the angle between the interelectronic distance vectors $\boldsymbol{u}$ and $\boldsymbol{v}$. By appropriate integration of $\Omega(u, v, \omega)$, we have created a family of intracules that provide information about various combinations of $u, v$, and $\omega^{10,12}$

\footnotetext{
a) Present address: Department of Chemistry, University of Prince Edward Island, Charlottetown PE, Canada C1A 4P3.

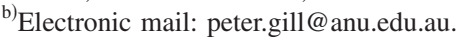

Parts II and III of this series ${ }^{13,14}$ have focused on the dot intracule $D(x)$, the quasidensity of finding two electrons with $\boldsymbol{u} \cdot \boldsymbol{v}=x$. This intracule offers a natural and efficient starting point for correlation energy calculations because it contains information about interelectronic positions and momenta and because its Fourier transform is available in closed form. ${ }^{14}$ Our electron correlation model is based on the primary conjecture that the correlation energy is a universal functional of $\Omega(u, v, \omega)$ and the secondary conjectures,

$$
\begin{aligned}
& E_{c}=\int_{0}^{\infty} \int_{0}^{\infty} \int_{0}^{\pi} \Omega(u, v, \omega) G(u, v, \omega) d \omega d v d u, \\
& E_{c}=\int_{-\infty}^{\infty} D(x) G(x) d x,
\end{aligned}
$$

where $G(u, v, \omega)$ and $G(x)$ are universal kernels.

Until now, our assessment of such intracule-based correlation models has been based entirely on intracules generated from $\mathrm{HF}$ wave functions expanded in the $6-311 \mathrm{G}$ basis set and we have assumed, perhaps optimistically, that this basis is sufficiently large that it has not introduced any artifacts into our results. In the present work, we address this issue directly by investigating the basis set dependence of $P(u)$ and $D(x)$ and exploring the extent to which basis set effects on $D(x)$ affect the correlation energies obtained from the dot ansatz (2). All calculations were performed using a locally modified version of the Q-CHEM quantum chemistry package $^{15}$ and we use atomic units throughout.

\section{BASIS SET EFFECTS ON INTRACULES}

If the wave function is expanded in a one-electron basis set $\left\{\chi_{\mu}\right\}$, an intracule $I$ can be expressed as

$$
I=\sum_{\mu \nu \lambda \sigma} \Gamma_{\mu \nu \lambda \sigma}[\mu \nu \lambda \sigma]_{I},
$$

where $\Gamma_{\mu \nu \lambda \sigma}$ is the two-particle density matrix ${ }^{16}$ and $[\mu \nu \lambda \sigma]_{I}$ are the associated intracule integrals. In the case of Gaussian basis functions, the position integrals $[s s s s]_{P}$ and dot integrals $[s s s]_{D}$ are straightforward ${ }^{12,13}$ and integrals of higher angular momentum can be found by differentiation with re- 

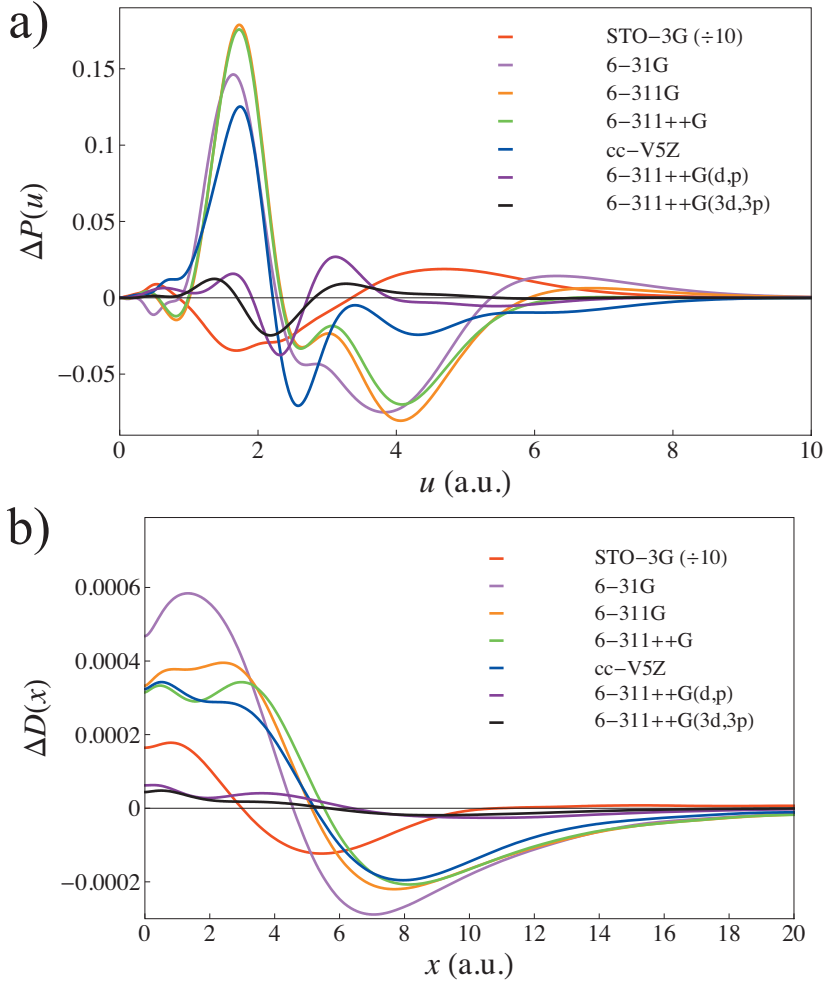

FIG. 1. (Color) Difference intracules for $\mathrm{CH}_{4}$ with respect to the $\mathrm{HF} / 6-311++\mathrm{G}(3 d f, 3 p d)$ reference in (a) position space and (b) dot space.

spect to the Cartesian centers of the basis functions, as first suggested by Boys. ${ }^{17}$

Position and dot intracules for the 18 atoms and 56 molecules of the G1 data set ${ }^{18}$ have been calculated from HF wave functions with Pople basis sets of increasing complexity. With the exception of $6-31 \mathrm{G}$, each member of the series $6-31 \mathrm{G}, 6-311 \mathrm{G}, 6-311++\mathrm{G}, 6-311++\mathrm{G}(d, p), 6-311+$ $+\mathrm{G}(3 d, 3 p)$, and $6-311++\mathrm{G}(3 d f, 3 p d)$ is a superset of the previous basis with additional diffuse or polarization functions, thus allowing us unambiguously to ascribe intracule differences to those modifications. In addition, we explored the STO-3G basis set as a low-level marker and the nonpolarized part of Dunning's cc-pV5Z basis set (here denoted cc-V5Z) ${ }^{19}$ as an indicator for how well the triple-splitvalence 6-311G set spans $s p$ space.

Taking the $6-311++\mathrm{G}(3 d f, 3 p d)$ intracules as a benchmark $\left(I_{\text {Ref }}\right)$, we have used the difference intracule

$$
\Delta I=I_{\text {Ref }}-I
$$

as a measure of basis set effect. To illustrate this, Fig. 1 shows several difference intracules for methane.

The trends in Figs. 1(a) and 1(b) are similar. As expected, STO-3G differences are much larger than the rest (note the scale of the STO-3G plots) and there is a marked decrease in error by going to 6-31G. A slight overall improvement is observed with the $6-311 \mathrm{G}$ basis yet the addition of diffuse functions does little to change the behavior of $\Delta P(u)$ or $\Delta D(x)$. We do, however, observe significant improvement upon inclusion of the polarization functions in the $6-311++\mathrm{G}(d, p)$ basis. The addition of further polarization functions yields only a slight improvement.
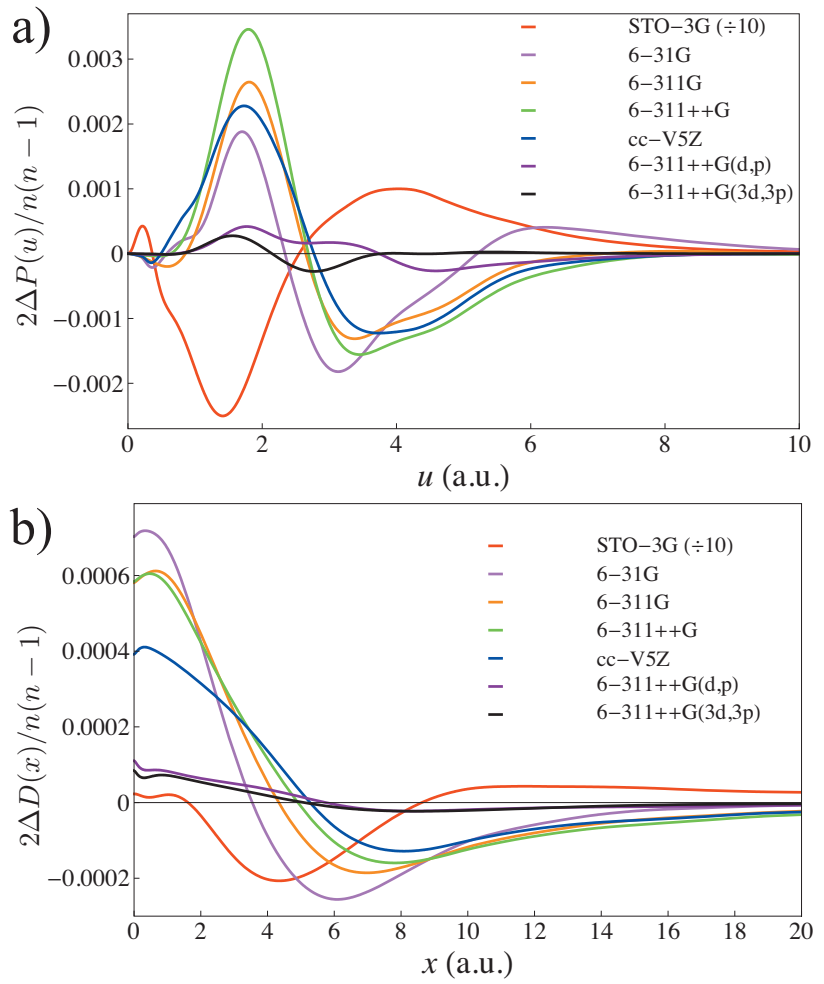

FIG. 2. (Color) Normalized average difference intracules for the G1 data set with respect to the $\mathrm{HF} / 6-311++\mathrm{G}(3 d f, 3 p d)$ reference in (a) position space and (b) dot space.

Because $\Delta I$ is proportional to the number of electron pairs $\left(n_{p}\right)$, we have plotted the renormalized difference intracule $(\Delta I / n p)$, averaged over all atoms and molecules in the G1 data set. These data, presented in Fig. 2 allow us to examine consistent trends in the shape of $\Delta P$ and $\Delta D$ across the data set. The similarity between Figs. 1 and 2 reveals that the gross basis set effects on the intracules of methane are also present in many other molecules. The persistence of this structure in the averaged difference intracules demonstrates consistent trends in basis set effects across the G1 data set.

We see from Fig. 2(a) that, when one moves from STO-3G to the large reference basis set, there is a reduction in the probability of finding electrons close together $(u$ $\approx 1.5$ ) and a corresponding increase in the likelihood of finding them further apart $(u \approx 4)$. One can think of such a change as a "basis hole," not unlike the "correlation hole" first discussed by Coulson and Neilson. ${ }^{1}$ Figure 2(b) reveals a comparable "basis hole" in the dot intracule.

An unexpected phenomenon arises, however, when we improve the quality of the $s p$ basis without adding polarization functions. The curves for $6-31 \mathrm{G}, 6-311 \mathrm{G}, 6-311++\mathrm{G}$, and cc-V5Z in Fig. 2(a) show that such bases overcompensate for the shortcomings of STO-3G and predict that electrons tend to be further apart than they are in the large reference basis. The resulting "basis antiholes" imply that the electrons move closer together when polarization functions are included and, although this is counterintuitive, it is confirmed by the observation that the electron repulsion energy $E_{\mathrm{J}}$ usually follows the trend 
TABLE I. $100 \Delta_{P}$ and $100 \Delta_{D}$ from various basis sets for the molecules in the G1 data set. The superscript indicates the basis set of the difference intracule from which $\Delta_{P}$ or $\Delta_{D}$ was calculated. (1) STO-3G, (2) 6-31G, (3) 6-311G, (4) 6-311++G, (5) cc-V5Z, (6) 6-311++G(d,p), and (7) 6-311++G(3d,3p).

\begin{tabular}{|c|c|c|c|c|c|c|c|c|c|c|c|c|c|c|}
\hline Molecule & $\Delta_{P}^{1}$ & $\Delta_{P}^{2}$ & $\Delta_{P}^{3}$ & $\Delta_{P}^{4}$ & $\Delta_{P}^{5}$ & $\Delta_{P}^{6}$ & $\Delta_{P}^{7}$ & $\Delta_{D}^{1}$ & $\Delta_{D}^{2}$ & $\Delta_{D}^{3}$ & $\Delta_{D}^{4}$ & $\Delta_{D}^{5}$ & $\Delta_{D}^{6}$ & $\Delta_{D}^{7}$ \\
\hline $\mathrm{H}$ & 0 & 0 & 0 & 0 & 0 & 0 & 0 & 0 & 0 & 0 & 0 & 0 & 0 & 0 \\
\hline $\mathrm{He}$ & 7.24 & 0.54 & 0 & 0 & 0.23 & 0 & 0 & 4.02 & 0.78 & 0 & 0 & 0.36 & 0 & 0 \\
\hline $\mathrm{Be}$ & 22.15 & 4.62 & 0.47 & 0 & 0.40 & 0 & 0 & 14.74 & 3.33 & 0.41 & 0 & 0.02 & 0 & 0 \\
\hline B & 25.00 & 4.43 & 0.69 & 0.12 & 0.63 & 0.18 & 0 & 13.98 & 2.20 & 0.30 & 0.41 & 0.31 & 0.10 & 0 \\
\hline $\mathrm{C}$ & 16.27 & 2.68 & 0.63 & 0.07 & 0.66 & 0.07 & 0 & 7.37 & 1.36 & 0.25 & 0.17 & 0.17 & 0.06 & 0 \\
\hline F & 13.51 & 1.62 & 0.90 & 0.03 & 0.83 & 0.01 & 0.02 & 5.49 & 0.56 & 0.35 & 0.07 & 0.25 & 0.01 & 0.02 \\
\hline $\mathrm{Ne}$ & 14.89 & 1.75 & 0.94 & 0 & 0.90 & 0 & 0 & 5.83 & 0.58 & 0.35 & 0 & 0.26 & 0 & 0 \\
\hline $\mathrm{Na}$ & 21.44 & 0.65 & 0.04 & 0 & 0.43 & 0 & 0 & 16.99 & 0.24 & 0.02 & 0 & 0.22 & 0 & 0 \\
\hline $\mathrm{Mg}$ & 23.15 & 0.33 & 0.07 & 0 & 0.20 & 0 & 0 & 17.83 & 0.10 & 0.04 & 0 & 0.08 & 0 & 0 \\
\hline $\mathrm{Al}$ & 21.53 & 0.22 & 0.09 & 0.15 & 0.12 & 0.10 & 0 & 16.27 & 0.11 & 0.12 & 0.17 & 0.19 & 0.06 & 0 \\
\hline $\mathrm{Ar}$ & 7.57 & 0.29 & 0.11 & 0 & 0.14 & 0 & 0 & 4.25 & 0.12 & 0.09 & 0 & 0.03 & 0 & 0 \\
\hline $\mathrm{H}_{2}$ & 4.66 & 2.50 & 1.86 & 1.86 & 1.70 & 0.21 & 0.02 & 4.40 & 2.67 & 1.99 & 1.95 & 1.81 & 0.13 & 0.02 \\
\hline $\mathrm{LiH}$ & 7.61 & 3.82 & 1.48 & 1.43 & 0.95 & 0.49 & 0.08 & 3.42 & 2.52 & 0.84 & 0.83 & 0.58 & 0.28 & 0.05 \\
\hline $\mathrm{BeH}$ & 11.52 & 2.28 & 1.14 & 0.93 & 2.52 & 0.16 & 0.10 & 5.19 & 0.92 & 0.51 & 0.49 & 8.32 & 0.07 & 0.05 \\
\hline $\mathrm{Li}_{2}$ & 9.89 & 1.79 & 0.42 & 0.36 & 0.36 & 0.04 & 0.05 & 6.70 & 1.09 & 0.18 & 0.15 & 0.19 & 0.10 & 0.09 \\
\hline $\mathrm{CH}$ & 9.19 & 1.31 & 0.88 & 0.86 & 0.65 & 0.11 & 0.04 & 4.34 & 1.04 & 0.56 & 0.57 & 0.43 & 0.06 & 0.02 \\
\hline $\mathrm{NH}$ & 6.86 & 0.74 & 0.80 & 0.90 & 0.53 & 0.11 & 0.01 & 5.04 & 0.83 & 0.66 & 0.53 & 0.39 & 0.09 & 0.01 \\
\hline $\mathrm{CH}_{2}\left({ }^{1} A_{1}\right)$ & 5.60 & 1.03 & 1.22 & 1.28 & 1.06 & 0.18 & 0.05 & 4.00 & 1.18 & 1.01 & 0.90 & 0.81 & 0.08 & 0.03 \\
\hline $\mathrm{CH}_{2}\left({ }^{3} B_{1}\right)$ & 6.20 & 1.01 & 0.69 & 0.63 & 0.60 & 0.13 & 0.09 & 3.83 & 0.74 & 0.51 & 0.47 & 0.39 & 0.08 & 0.06 \\
\hline $\mathrm{OH}$ & 8.31 & 0.97 & 0.85 & 0.85 & 0.44 & 0.10 & 0.04 & 5.85 & 0.87 & 0.63 & 0.47 & 0.30 & 0.06 & 0.04 \\
\hline $\mathrm{NH}_{2}$ & 4.92 & 1.08 & 1.09 & 1.25 & 0.85 & 0.13 & 0.01 & 4.62 & 1.19 & 1.03 & 0.80 & 0.69 & 0.08 & 0.02 \\
\hline $\mathrm{CO}$ & 3.99 & 2.18 & 1.96 & 1.95 & 1.61 & 0.22 & 0.16 & 3.03 & 1.68 & 1.58 & 1.52 & 1.32 & 0.28 & 0.22 \\
\hline $\mathrm{HCN}$ & 3.46 & 1.33 & 1.34 & 1.43 & 1.28 & 0.21 & 0.14 & 2.62 & 1.47 & 1.42 & 1.36 & 1.26 & 0.22 & 0.20 \\
\hline $\mathrm{C}_{2} \mathrm{H}_{2}$ & 4.23 & 1.19 & 0.85 & 0.82 & 0.81 & 0.22 & 0.19 & 2.09 & 1.08 & 1.02 & 1.01 & 0.94 & 0.21 & 0.20 \\
\hline $\mathrm{NO}$ & 3.71 & 2.08 & 1.80 & 1.87 & 1.43 & 0.29 & 0.14 & 3.67 & 1.79 & 1.66 & 1.68 & 1.30 & 0.23 & 0.22 \\
\hline $\mathrm{HCO}$ & 3.47 & 1.35 & 1.33 & 1.37 & 1.08 & 0.16 & 0.14 & 3.58 & 1.40 & 1.32 & 1.19 & 1.04 & 0.19 & 0.20 \\
\hline $\mathrm{SiH}_{2}\left({ }^{1} A_{1}\right)$ & 6.99 & 1.50 & 1.53 & 1.53 & 1.41 & 0.17 & 0.09 & 4.14 & 1.16 & 1.03 & 1.07 & 1.02 & 0.08 & 0.02 \\
\hline $\mathrm{SiH}_{2}\left({ }^{3} B_{1}\right)$ & 8.42 & 1.12 & 1.13 & 1.06 & 1.96 & 0.10 & 0.09 & 5.07 & 0.81 & 0.71 & 0.72 & 0.96 & 0.06 & 0.03 \\
\hline $\mathrm{O}_{2}$ & 4.32 & 1.86 & 1.52 & 1.68 & 1.15 & 0.43 & 0.15 & 3.52 & 1.51 & 1.28 & 1.40 & 1.22 & 0.25 & 0.26 \\
\hline $\mathrm{H}_{2} \mathrm{CO}$ & 2.34 & 1.03 & 1.04 & 1.12 & 0.85 & 0.15 & 0.12 & 3.22 & 1.41 & 1.32 & 1.16 & 1.02 & 0.24 & 0.26 \\
\hline $\mathrm{C}_{2} \mathrm{H}_{4}$ & 2.47 & 0.58 & 0.55 & 0.61 & 0.56 & 0.14 & 0.10 & 1.74 & 0.66 & 0.65 & 0.65 & 0.59 & 0.21 & 0.19 \\
\hline $\mathrm{PH}_{2}$ & 6.14 & 1.35 & 1.39 & 1.38 & 1.28 & 0.19 & 0.10 & 4.15 & 1.01 & 1.02 & 0.98 & 0.90 & 0.10 & 0.04 \\
\hline $\mathrm{SiH}_{3}$ & 5.84 & 1.41 & 1.51 & 1.49 & 1.29 & 0.13 & 0.12 & 3.26 & 1.16 & 1.04 & 1.06 & 1.00 & 0.11 & 0.04 \\
\hline $\mathrm{HCl}$ & 5.84 & 0.69 & 0.73 & 0.65 & 0.67 & 0.08 & 0.07 & 4.02 & 0.42 & 0.51 & 0.46 & 0.39 & 0.06 & 0.03 \\
\hline $\mathrm{H}_{2} \mathrm{~S}$ & 5.65 & 1.24 & 1.25 & 1.24 & 1.09 & 0.15 & 0.12 & 4.30 & 0.88 & 0.95 & 0.91 & 0.80 & 0.11 & 0.05 \\
\hline $\mathrm{PH}_{3}$ & 4.29 & 1.63 & 1.67 & 1.65 & 1.52 & 0.23 & 0.13 & 3.98 & 1.31 & 1.29 & 1.26 & 1.18 & 0.14 & 0.04 \\
\hline $\mathrm{SiH}_{4}$ & 4.02 & 1.68 & 1.86 & 1.81 & 1.58 & 0.17 & 0.14 & 3.10 & 1.48 & 1.36 & 1.36 & 1.29 & 0.12 & 0.05 \\
\hline $\mathrm{F}_{2}$ & 5.65 & 0.64 & 0.85 & 0.91 & 0.60 & 0.42 & 0.19 & 4.08 & 0.91 & 0.86 & 0.89 & 0.61 & 0.46 & 0.33 \\
\hline $\mathrm{H}_{2} \mathrm{O}_{2}$ & 3.26 & 1.04 & 0.93 & 1.06 & 0.69 & 0.32 & 0.12 & 4.26 & 1.39 & 1.20 & 1.12 & 0.89 & 0.34 & 0.21 \\
\hline $\mathrm{N}_{2} \mathrm{H}_{4}$ & 2.12 & 0.93 & 0.78 & 0.74 & 0.57 & 0.15 & 0.05 & 2.91 & 1.29 & 1.05 & 0.94 & 0.80 & 0.23 & 0.15 \\
\hline $\mathrm{H}_{3} \mathrm{COH}$ & 1.83 & 0.67 & 0.67 & 0.69 & 0.53 & 0.13 & 0.07 & 3.17 & 1.13 & 0.98 & 0.79 & 0.68 & 0.21 & 0.20 \\
\hline $\mathrm{C}_{2} \mathrm{H}_{6}$ & 1.39 & 0.61 & 0.63 & 0.61 & 0.58 & 0.11 & 0.05 & 1.43 & 0.59 & 0.45 & 0.42 & 0.33 & 0.12 & 0.10 \\
\hline $\mathrm{CO}_{2}$ & 3.42 & 2.12 & 2.01 & 2.05 & 1.92 & 0.28 & 0.23 & 2.60 & 1.60 & 1.57 & 1.54 & 1.40 & 0.31 & 0.44 \\
\hline
\end{tabular}


TABLE I. (Continued.)

\begin{tabular}{|c|c|c|c|c|c|c|c|c|c|c|c|c|c|c|}
\hline Molecule & $\Delta_{P}^{1}$ & $\Delta_{P}^{2}$ & $\Delta_{P}^{3}$ & $\Delta_{P}^{4}$ & $\Delta_{P}^{5}$ & $\Delta_{P}^{6}$ & $\Delta_{P}^{7}$ & $\Delta_{D}^{1}$ & $\Delta_{D}^{2}$ & $\Delta_{D}^{3}$ & $\Delta_{D}^{4}$ & $\Delta_{D}^{5}$ & $\Delta_{D}^{6}$ & $\Delta_{D}^{7}$ \\
\hline $\mathrm{Na}_{2}$ & 9.54 & 0.44 & 0.18 & 0.14 & 0.23 & 0.11 & 0.02 & 9.01 & 0.31 & 0.07 & 0.08 & 0.14 & 0.03 & 0.01 \\
\hline $\mathrm{SiO}$ & 4.68 & 1.87 & 1.81 & 2.01 & 1.80 & 0.36 & 0.17 & 3.56 & 1.70 & 1.43 & 1.45 & 1.33 & 0.23 & 0.11 \\
\hline SO & 4.34 & 1.57 & 1.53 & 1.63 & 1.43 & 0.36 & 0.24 & 3.72 & 1.35 & 1.29 & 1.26 & 1.12 & 0.23 & 0.19 \\
\hline $\mathrm{ClO}$ & 2.93 & 1.25 & 1.22 & 1.17 & 0.99 & 0.35 & 0.23 & 3.58 & 0.98 & 0.99 & 0.96 & 0.84 & 0.26 & 0.20 \\
\hline $\mathrm{ClF}$ & 2.84 & 0.89 & 0.93 & 0.91 & 0.80 & 0.36 & 0.19 & 3.61 & 0.75 & 0.78 & 0.75 & 0.64 & 0.31 & 0.27 \\
\hline $\mathrm{HOCl}$ & 2.71 & 0.86 & 0.90 & 0.86 & 0.70 & 0.31 & 0.17 & 3.39 & 0.84 & 0.84 & 0.79 & 0.68 & 0.26 & 0.16 \\
\hline $\mathrm{CH}_{3} \mathrm{Cl}$ & 2.75 & 0.69 & 0.69 & 0.62 & 0.56 & 0.12 & 0.07 & 2.63 & 0.72 & 0.65 & 0.63 & 0.54 & 0.21 & 0.16 \\
\hline $\mathrm{CH}_{3} \mathrm{SH}$ & 2.75 & 0.84 & 0.88 & 0.85 & 0.71 & 0.12 & 0.08 & 2.82 & 0.83 & 0.80 & 0.77 & 0.67 & 0.20 & 0.15 \\
\hline $\mathrm{NaCl}$ & 4.47 & 0.44 & 0.65 & 0.52 & 0.44 & 0.13 & 0.08 & 2.85 & 0.19 & 0.45 & 0.36 & 0.25 & 0.08 & 0.04 \\
\hline $\mathrm{Si}_{2}$ & 7.42 & 2.84 & 1.95 & 1.61 & 2.77 & 0.73 & 0.12 & 3.13 & 0.77 & 0.51 & 1.03 & 0.75 & 0.29 & 0.05 \\
\hline $\mathrm{P}_{2}$ & 5.46 & 2.01 & 2.20 & 2.09 & 1.92 & 0.64 & 0.23 & 2.81 & 1.46 & 1.50 & 1.47 & 1.36 & 0.37 & 0.18 \\
\hline $\mathrm{S}_{2}$ & 4.69 & 1.85 & 1.68 & 1.60 & 1.53 & 0.51 & 0.29 & 2.87 & 1.25 & 1.20 & 1.17 & 1.09 & 0.33 & 0.24 \\
\hline $\mathrm{SO}_{2}$ & 2.95 & 2.16 & 2.19 & 2.25 & 1.95 & 0.51 & 0.26 & 3.47 & 2.04 & 1.91 & 1.91 & 1.73 & 0.39 & 0.34 \\
\hline $\mathrm{Cl}_{2}$ & 2.89 & 1.25 & 1.28 & 1.09 & 0.85 & 0.40 & 0.29 & 3.04 & 0.85 & 0.81 & 0.77 & 0.70 & 0.26 & 0.17 \\
\hline $\mathrm{Si}_{2} \mathrm{H}_{6}$ & 2.30 & 1.63 & 1.68 & 1.66 & 1.50 & 0.11 & 0.07 & 2.95 & 0.79 & 0.77 & 0.76 & 0.72 & 0.19 & 0.15 \\
\hline \multicolumn{15}{|c|}{ Mean absolute errors } \\
\hline Atoms only & 14.86 & 1.31 & 0.37 & 0.03 & 0.46 & 0.03 & 0.01 & 9.39 & 0.75 & 0.18 & 0.07 & 0.16 & 0.02 & 0.01 \\
\hline $\begin{array}{l}\text { Molecules } \\
\text { only }\end{array}$ & 4.95 & 1.39 & 1.21 & 1.19 & 1.08 & 0.23 & 0.12 & 3.80 & 1.16 & 0.99 & 0.95 & 0.98 & 0.18 & 0.13 \\
\hline $\begin{array}{l}\text { Atoms and } \\
\text { molecules }\end{array}$ & 7.36 & 1.37 & 1.01 & 0.91 & 0.93 & 0.18 & 0.09 & 5.18 & 1.06 & 0.79 & 0.73 & 0.78 & 0.14 & 0.10 \\
\hline
\end{tabular}

$$
\begin{aligned}
E_{J}[\mathrm{STO}-3 \mathrm{G}] & >E_{J}[6-311++\mathrm{G}(3 d f, 3 p d)] \\
& >E_{J}[6-31 \mathrm{G}] .
\end{aligned}
$$

The trend in $E_{J}$ for a given basis (i.e., $E_{J}[6-311+$ $+\mathrm{G}(3 d f, 3 p d)]-E_{J}[$ Basis $\left.]=\Delta E_{J}\right)$ is also exactly determined from the difference intracules by the relation

$$
\Delta E_{J}+\Delta E_{K}=\int_{0}^{\infty} \Delta P(u) u^{-1} d u,
$$

which emphasizes the importance of the short-range part of the intracule in determining the repulsion energy. Thus, a basis hole is indicative of a positive deviation in $E_{J}$ from the reference and a basis antihole is indicative of a negative deviation.

Of course, this effect is more than counterbalanced by the nuclear attraction energy and the total energy of the $6-311++\mathrm{G}(3 d f, 3 p d)$ wave function is lower than that of the 6-31G wave function, despite the electrons in the former tending to be closer together. Such an effect is reminiscent of the secondary Coulomb hole recently observed ${ }^{4}$ in the helium atom, wherein correlation is found to reduce $P(u)$ for large $u$.

The polarization functions in $6-311++\mathrm{G}(d, p)$ largely eliminate the basis antihole and yield position and dot intracules that are probably very close to the HF limit.

It is convenient to be able to reduce $\Delta I$ to a single number that measures the overall faithfulness of the intracule to its benchmark. Because our intracules are always normalized to the number $n(n-1) / 2$ of pairs of electrons, the integral of $\Delta I$ vanishes identically. However, the integral of $|\Delta I|$ is nonnegative and the renormalized quantities,

$$
\Delta_{P}=\frac{2}{n(n-1)} \int_{0}^{\infty}|\Delta P(u)| d u,
$$

$$
\Delta_{D}=\frac{2}{n(n-1)} \int_{-\infty}^{\infty}|\Delta D(x)| d x,
$$

provide useful measures of the deviation of an intracule from its benchmark. We have also explored the use of the maximum difference between intracules to quantify basis set effects but have found that the trends in this quantity are very similar to those for $\Delta_{P}$ and $\Delta_{D}$. The $\Delta_{P}$ and $\Delta_{D}$ values for each basis set and each atom or molecule in the G1 data set are listed in Table I.

The results in Table I reinforce the qualitative conclusions drawn from Figs. 1 and 2. The greatest improvement in the quality of the intracule is achieved in going from STO-3G (which is particularly poor for atoms) to 6-31G. After this, the next most significant improvement comes from the addition of the first set of polarization functions to the triple-split-valence $6-311 \mathrm{G}$ and only a very slight improvement is obtained from the higher polarization functions. Again, diffuse functions do not appear to have any significant effect on the quality of the intracule, although it should be noted that there are no anionic systems in the G1 data set.

The cc-V5Z basis (which includes only $s$ and $p$ functions for heavy atoms and $s$ functions for $\mathrm{H}$ and $\mathrm{He}$ ) appears similar to $6-31 \mathrm{G}, 6-311 \mathrm{G}$, and $6-311++\mathrm{G}$, indicating that the effect of additional valence functions beyond those in $6-311++\mathrm{G}$ is modest.

\section{BASIS SET EFFECTS ON CORRELATION ENERGIES}

A correlation model based on the dot intracule ansatz (2) requires a kernel and we have explored ${ }^{13,14}$ 
TABLE II. The exact correlation energy $E_{c}^{\text {exact }}$ and errors $\Delta E_{c}^{\text {Basis }}$ from various basis sets for the molecules in the G1 data set. All values in $\mathrm{m} E_{h}$. (1) STO-3G, (2) 6-31G, (3) 6-311G, (4) 6-311++G, (5) cc-V5Z, (6) 6-311++G(d,p), (7) 6-311++G(3d,3p), and (8) 6-311++G(3df,3pd).

\begin{tabular}{|c|c|c|c|c|c|c|c|c|c|}
\hline Molecule & $-E_{c}^{\text {exact }}$ & $\Delta E_{c}^{1}$ & $\Delta E_{c}^{2}$ & $\Delta E_{c}^{3}$ & $\Delta E_{c}^{4}$ & $\Delta E_{c}^{5}$ & $\Delta E_{c}^{6}$ & $\Delta E_{c}^{7}$ & $\Delta E_{c}^{8}$ \\
\hline $\mathrm{H}$ & 0 & 0 & 0 & 0 & 0 & 0 & 0 & 0 & 0 \\
\hline $\mathrm{He}$ & 42 & 0 & 1 & 1 & 1 & 1 & 1 & 1 & 1 \\
\hline $\mathrm{Li}$ & 45 & 1 & 0 & 0 & 0 & 1 & 0 & 0 & 0 \\
\hline $\mathrm{Be}$ & 94 & -12 & -13 & -12 & -12 & -12 & -12 & -12 & -12 \\
\hline B & 121 & -11 & -10 & -8 & -8 & -8 & -9 & -9 & -9 \\
\hline $\mathrm{C}$ & 151 & -5 & -1 & 0 & 1 & 0 & 0 & 0 & 0 \\
\hline $\mathrm{N}$ & 185 & 5 & 13 & 14 & 14 & 14 & 14 & 14 & 14 \\
\hline $\mathrm{O}$ & 249 & -3 & 5 & 5 & 5 & 4 & 4 & 4 & 4 \\
\hline $\mathrm{F}$ & 318 & -8 & -1 & -1 & -1 & -1 & -1 & -1 & -1 \\
\hline $\mathrm{Ne}$ & 391 & -9 & -2 & -2 & -2 & -2 & -2 & -2 & -2 \\
\hline $\mathrm{Na}$ & 396 & -1 & -6 & -8 & -8 & -7 & -8 & -8 & -8 \\
\hline $\mathrm{Mg}$ & 438 & -17 & -14 & -14 & -14 & -14 & -14 & -14 & -14 \\
\hline $\mathrm{Al}$ & 465 & -16 & -11 & -11 & -11 & -11 & -12 & -12 & -12 \\
\hline $\mathrm{Si}$ & 500 & -17 & -9 & -9 & -9 & -9 & -10 & -10 & -10 \\
\hline $\mathrm{P}$ & 540 & -17 & -5 & -1 & -3 & -4 & -3 & -3 & -3 \\
\hline S & 597 & -14 & 0 & 1 & 0 & 0 & -1 & -1 & -1 \\
\hline $\mathrm{Cl}$ & 658 & -11 & 7 & 9 & 8 & 7 & 7 & 7 & 7 \\
\hline $\mathrm{Ar}$ & 723 & -3 & 18 & 18 & 18 & 18 & 18 & 18 & 18 \\
\hline $\mathrm{H}_{2}$ & 41 & -2 & -1 & -1 & -1 & -1 & -1 & -1 & -1 \\
\hline $\mathrm{LiH}$ & 83 & 6 & 6 & 7 & 7 & 7 & 7 & 7 & 7 \\
\hline $\mathrm{BeH}$ & 93 & 5 & 5 & 5 & 6 & 14 & 6 & 6 & 6 \\
\hline $\mathrm{Li}_{2}$ & 124 & 4 & 4 & 4 & 5 & 5 & 5 & 5 & 5 \\
\hline $\mathrm{CH}$ & 194 & -7 & -4 & -3 & -3 & -3 & -3 & -3 & -3 \\
\hline $\mathrm{NH}$ & 236 & 5 & 13 & 13 & 14 & 14 & 14 & 14 & 14 \\
\hline $\mathrm{CH}_{2}\left({ }^{1} A_{1}\right)$ & 239 & -7 & -4 & -4 & -4 & -4 & -4 & -4 & -4 \\
\hline $\mathrm{CH}_{2}\left({ }^{3} B_{1}\right)$ & 208 & 23 & 30 & 31 & 32 & 31 & 32 & 32 & 32 \\
\hline $\mathrm{OH}$ & 309 & -5 & 3 & 4 & 4 & 4 & 5 & 5 & 5 \\
\hline $\mathrm{NH}_{2}$ & 287 & 13 & 20 & 20 & 21 & 20 & 21 & 21 & 21 \\
\hline $\mathrm{CH}_{3}$ & 254 & 38 & 45 & 45 & 46 & 45 & 45 & 45 & 45 \\
\hline $\mathrm{HF}$ & 389 & -16 & -5 & -4 & -4 & -4 & -3 & -3 & -3 \\
\hline $\mathrm{H}_{2} \mathrm{O}$ & 371 & -2 & 8 & 9 & 10 & 9 & 11 & 11 & 11 \\
\hline $\mathrm{NH}_{3}$ & 340 & 26 & 31 & 32 & 33 & 32 & 33 & 33 & 33 \\
\hline $\mathrm{CH}_{4}$ & 299 & 55 & 60 & 60 & 60 & 60 & 59 & 59 & 59 \\
\hline $\mathrm{LiF}$ & 441 & -23 & -4 & -3 & -2 & -2 & -2 & -2 & -2 \\
\hline $\mathrm{CN}$ & 483 & -66 & -63 & -63 & -63 & -63 & -58 & -58 & -58 \\
\hline $\mathrm{N}_{2}$ & 549 & -61 & -59 & -58 & -58 & -59 & -57 & -57 & -57 \\
\hline $\mathrm{CO}$ & 535 & -48 & -46 & -45 & -45 & -45 & -45 & -45 & -44 \\
\hline $\mathrm{HCN}$ & 515 & -26 & -23 & -23 & -23 & -23 & -22 & -22 & -22 \\
\hline $\mathrm{C}_{2} \mathrm{H}_{4}$ & 480 & 11 & 14 & 14 & 14 & 14 & 14 & 14 & 14 \\
\hline NO & 596 & -69 & -63 & -63 & -64 & -62 & -61 & -61 & -61 \\
\hline $\mathrm{HCO}$ & 553 & -34 & -26 & -26 & -26 & -26 & -25 & -25 & -25 \\
\hline $\mathrm{SiH}_{2}\left({ }^{1} A_{1}\right)$ & 567 & -2 & 7 & 7 & 7 & 7 & 7 & 6 & 6 \\
\hline $\mathrm{SiH}_{2}\left({ }^{3} B_{1}\right)$ & 540 & 18 & 28 & 28 & 28 & 28 & 27 & 26 & 25 \\
\hline $\mathrm{O}_{2}$ & 636 & -59 & -54 & -54 & -55 & -56 & -54 & -54 & -54 \\
\hline $\mathrm{H}_{2} \mathrm{CO}$ & 586 & -17 & -12 & -12 & -12 & -12 & -12 & -12 & -12 \\
\hline $\mathrm{C}_{2} \mathrm{H}_{4}$ & 518 & 49 & 54 & 54 & 53 & 52 & 53 & 52 & 53 \\
\hline $\mathrm{PH}_{2}$ & 611 & 14 & 30 & 30 & 29 & 29 & 29 & 29 & 29 \\
\hline $\mathrm{SiH}_{3}$ & 575 & 34 & 45 & 45 & 45 & 45 & 40 & 39 & 38 \\
\hline $\mathrm{HCl}$ & 707 & 4 & 24 & 24 & 24 & 23 & 25 & 25 & 25 \\
\hline $\mathrm{H}_{2} \mathrm{~S}$ & 683 & 14 & 33 & 31 & 32 & 32 & 33 & 33 & 33 \\
\hline $\mathrm{PH}_{3}$ & 652 & 27 & 43 & 42 & 42 & 42 & 41 & 41 & 41 \\
\hline $\mathrm{SiH}_{4}$ & 606 & 53 & 63 & 62 & 62 & 63 & 55 & 53 & 53 \\
\hline $\mathrm{F}_{2}$ & 757 & -83 & -75 & -74 & -76 & -77 & -74 & -74 & -73 \\
\hline $\mathrm{H}_{2} \mathrm{O}_{2}$ & 711 & -43 & -31 & -30 & -32 & -33 & -30 & -30 & -30 \\
\hline $\mathrm{N}_{2} \mathrm{H}_{4}$ & 641 & 25 & 33 & 34 & 32 & 31 & 33 & 33 & 33 \\
\hline $\mathrm{H}_{3} \mathrm{COH}$ & 629 & 29 & 38 & 38 & 37 & 36 & 37 & 37 & 37 \\
\hline $\mathrm{C}_{2} \mathrm{H}_{6}$ & 561 & 91 & 96 & 96 & 95 & 94 & 94 & 94 & 94 \\
\hline $\mathrm{CO}_{2}$ & 876 & -59 & -53 & -52 & -51 & -52 & -49 & -50 & -50 \\
\hline $\mathrm{CS}$ & 867 & -47 & -34 & -33 & -33 & -33 & -31 & -31 & -30 \\
\hline
\end{tabular}


TABLE II. (Continued.)

\begin{tabular}{|c|c|c|c|c|c|c|c|c|c|}
\hline Molecule & $-E_{c}^{\text {exact }}$ & $\Delta E_{c}^{1}$ & $\Delta E_{c}^{2}$ & $\Delta E_{c}^{3}$ & $\Delta E_{c}^{4}$ & $\Delta E_{c}^{5}$ & $\Delta E_{c}^{6}$ & $\Delta E_{c}^{7}$ & $\Delta E_{c}^{8}$ \\
\hline $\mathrm{Na}_{2}$ & 819 & -8 & -6 & -9 & -8 & -6 & -8 & -8 & -8 \\
\hline $\mathrm{SiO}$ & 879 & -61 & -49 & -48 & -46 & -46 & -46 & -45 & -45 \\
\hline SO & 957 & -48 & -34 & -33 & -34 & -35 & -31 & -32 & -32 \\
\hline $\mathrm{ClO}$ & 1002 & -46 & -28 & -28 & -29 & -30 & -27 & -26 & -26 \\
\hline $\mathrm{ClF}$ & 1063 & -52 & -34 & -34 & -36 & -36 & -33 & -33 & -33 \\
\hline $\mathrm{HOCl}$ & 1045 & -32 & -14 & -13 & -14 & -15 & -11 & -11 & -11 \\
\hline $\mathrm{CH}_{3} \mathrm{Cl}$ & 968 & 42 & 59 & 58 & 58 & 57 & 58 & 58 & 58 \\
\hline $\mathrm{CH}_{3} \mathrm{SH}$ & 946 & 53 & 69 & 68 & 68 & 67 & 68 & 68 & 68 \\
\hline $\mathrm{NaCl}$ & 1101 & 12 & 30 & 31 & 30 & 31 & 31 & 31 & 31 \\
\hline $\mathrm{Si}_{2}$ & 1077 & -53 & -19 & -29 & -39 & -20 & -39 & -38 & -38 \\
\hline $\mathrm{P}_{2}$ & 1205 & -45 & -24 & -21 & -22 & -24 & -18 & -17 & -17 \\
\hline $\mathrm{S}_{2}$ & 1275 & -19 & 4 & 2 & 3 & 2 & 6 & 7 & 7 \\
\hline $\mathrm{SO}_{2}$ & 1334 & -96 & -78 & -76 & -76 & -78 & -73 & -74 & -74 \\
\hline $\mathrm{Cl}_{2}$ & 1380 & -17 & 11 & 9 & 10 & 8 & 14 & 15 & 15 \\
\hline $\mathrm{Si}_{2} \mathrm{H}_{6}$ & 1183 & 97 & 117 & 113 & 113 & 114 & 100 & 98 & 97 \\
\hline \multicolumn{10}{|c|}{ Mean absolute errors } \\
\hline Atoms only & & 8 & 6 & 6 & 6 & 6 & 6 & 6 & 6 \\
\hline Molecules only & & 34 & 33 & 33 & 34 & 33 & 32 & 32 & 32 \\
\hline $\begin{array}{l}\text { Atoms and } \\
\text { molecules }\end{array}$ & & 28 & 27 & 26 & 27 & 26 & 26 & 26 & 26 \\
\hline
\end{tabular}

$$
G(x)=c_{D} \cos k_{0} x,
$$

where $c_{D}=-76.95 \mathrm{~m} E_{h}$ and $k_{0}=0.8474$ are parameters that have been optimized by fitting to the correlation energies of the first 18 atoms in the Periodic Table.

Table II lists the exact correlation energies ${ }^{20}$ for each system in the G1 data set as well as errors,

$$
\Delta E_{c}^{n}=E_{c}^{\text {exact }}-E_{c}^{n},
$$

in the correlation energies predicted from Eqs. (2) and (8), where the index $n$ denotes which basis set has been used, in order of increasing polarization and size.

Examination of Table II reveals that errors in the correlation energies calculated from Eqs. (2) and (8) are remarkably insensitive to the basis set used. The mean absolute error (MAE) for the full test set changes by only $2 \mathrm{~m} E_{h}$ between the minimal STO-3G basis and the large 6-311+ $+\mathrm{G}(3 d f, 3 p d)$ basis. We are relieved to find that our historical use of the triple-split-valence 6-311G basis appears to be justified.

The results in Table II were generated using parameters that were optimized using the $6-311 \mathrm{G}$ basis set. Alternatively, one may reoptimize $c_{D}$ and $k_{0}$ for each basis set by

TABLE III. Reoptimized parameters for each basis and MAE for the first 18 atoms of the periodic table. $c_{D}$ and MAE are given in $\mathrm{m} E_{h}$.

\begin{tabular}{lccc}
\hline \hline Basis set & $c_{D}$ & $k_{0}$ & MAE \\
\hline (1) STO-3G & -76.6 & 0.8405 & 5.1 \\
(2) 6-31G & -77.0 & 0.8473 & 6.4 \\
(3) 6-311G & -77.0 & 0.8474 & 6.4 \\
(4) 6-311++G & -77.0 & 0.8475 & 6.4 \\
(5) cc-V5Z & -77.1 & 0.8475 & 6.3 \\
(6) 6-311++G(d,p) & -77.1 & 0.8475 & 6.4 \\
(7) $6-311++\mathrm{G}(3 d, 3 p)$ & -77.1 & 0.8475 & 6.4 \\
(8) 6-311++G(3df,3pd) & -77.1 & 0.8475 & 6.7 \\
\hline
\end{tabular}

rms fitting the correlation energies of the first 18 atoms, as was done previously for the $6-311 \mathrm{G}$ basis. ${ }^{13}$ This yields the lowest possible rms error for a given basis.

Table III lists the reoptimized parameters $c_{D}$ and $k_{0}$ for each basis as well as MAE in the $E_{c}$ calculated for the first 18 atoms of the periodic table. These results show that $c_{D}$ and $k_{0}$ are also insensitive to the choice of basis. The MAE data also demonstrate that the correlation energies calculated with parameters optimized for the particular basis will not significantly improve the accuracy of the calculation.

\section{CONCLUDING REMARKS}

We have calculated position and dot intracules for the first 18 atoms of the periodic table and all 56 molecules of the G1 data set using a series of Pople basis sets and the nonpolarized part of Dunning's cc-pV5Z basis to determine the effect of basis set on intracule shape and the sensitivity of correlation energies based on intracule functional theory.

Using $\Delta_{P}$ and $\Delta_{D}$ as a measure, we have shown that the difference between the low-level STO-3G intracules and the reference $6-311++\mathrm{G}(3 d f, 3 p d)$ intracules is small but can be reduced further by using the triple-split-valence 6-311G basis. We have also shown that diffuse functions do not significantly change the intracules although we note that there are no anionic systems in our test set. In addition, we have observed that polarization functions improve the intracules only slightly.

The correlation energy is even less sensitive to the basis set than the intracules are. We have demonstrated that $E_{c}$ calculated using the dot ansatz is highly robust and we observe a $2 \mathrm{~m} E_{h}$ difference between the STO-3G and 6-311 $++\mathrm{G}(3 d f, 3 p d)$ mean absolute deviations across the entire test set of atoms and molecules.

Such observations provide an a posteriori justification 
for the use of HF/6-311G wave functions in intracule functional theory.

\section{ACKNOWLEDGMENTS}

P.M.W.G. thanks the APAC Merit Allocation Scheme for a generous grant of supercomputer resources and the Australian Research Council (Grant Nos. DP0664466 and DR0771978) for funding.

We thank Yves Bernard for helpful discussions regarding the correlation kernel for the dot intracule.

${ }^{1}$ C. A. Coulson and A. H. Neilson, Proc. Phys. Soc. London 78, 831 (1961).

2 A. M. Lee and P. M. W. Gill, Chem. Phys. Lett. 313, 271 (1999).

${ }^{3}$ D. P. O’Neill and P. M. W. Gill, Phys. Rev. A 68, 022505 (2003).

${ }^{4}$ J. K. Pearson, P. M. W. Gill, J. M. Ugalde, and R. J. Boyd, Mol. Phys. (unpublished).

${ }^{5}$ K. E. Banyard and C. E. Reed, J. Phys. B 11, 2957 (1978).

${ }^{6}$ N. A. Besley, A. M. Lee, and P. M. W. Gill, Mol. Phys. 100, 1763 (2002).

${ }^{7}$ V. A. Rassolov, J. Chem. Phys. 110, 3672 (1999).

${ }^{8}$ E. Wigner, Phys. Rev. 40, 749 (1932).

${ }^{9}$ H. J. Groenewold, Physica 12, 405 (1946).

${ }^{10}$ P. M. W. Gill, D. L. Crittenden, D. P. O’Neill, and N. A. Besley, Phys. Chem. Chem. Phys. 8, 15 (2006)
${ }^{11}$ D. L. Crittenden and P. M. W. Gill, J. Chem. Phys. 127, 014101 (2007).

${ }^{12}$ P. M. W. Gill, D. P. O’Neill, and N. A. Besley, Theor. Chem. Acc. 109, 241 (2003).

${ }^{13}$ D. L. Crittenden, E. E. Dumont, and P. M. W. Gill, J. Chem. Phys. 127, 141103 (2007).

${ }^{14}$ Y. A. Bernard, D. L. Crittenden, and P. M. W. Gill, Phys. Chem. Chem. Phys. 10, 3447 (2008).

${ }^{15}$ Y. Shao, L. Fusti-Molnar, Y. Jung, J. Kussmann, C. Ochsenfeld, S. T. Brown, A. T. B. Gilbert, L. V. Slipchenko, S. V. Levchenko, D. P. O’Neill, R. A. Distasio, Jr., R. C. Lochan, T. Wang, G. J. O. Beran, N. A. Besley, J. M. Herbert, C. Y. Lin, T. van Voorhis, S. H. Chien, A. Sodt, R. P. Steele, V. A. Rassolov, P. E. Maslen, P. P. Korambath, R. D. Adamson, B. Austin, J. Baker, E. F. C. Byrd, H. Dachsel, R. J. Doerksen, A. Dreuw, B. D. Dunietz, A. D. Dutoi, T. R. Furlani, S. R. Gwaltney, A. Heyden, S. Hirata, C.-P. Hsu, G. Kedziora, R. Z. Khalliulin, P. Klunzinger, A. M. Lee, M. S. Lee, W. Liang, I. Lotan, N. Nair, B. Peters, E. I. Proynov, P. A. Pieniazek, Y. M. Rhee, J. Ritchie, E. Rosta, C. D. Sherrill, A. C. Simmonett, J. E. Subotnik, H. L. Woodcock III, W. Zhang, A. T. Bell, A. K. Chakraborty, D. M. Chipman, F. J. Keil, A. Warshel, W. J. Hehre, H. F. Schaefer III, J. Kong, A. I. Krylov, P. M. W. Gill, and M. HeadGordon, Phys. Chem. Chem. Phys. 8, 3172 (2006).

${ }^{16}$ E. R. Davidson, Reduced Density Matrices in Quantum Chemistry (Academic, New York, 1976).

${ }^{17}$ S. F. Boys, Proc. R. Soc. London A200, 542 (1950).

${ }^{18}$ J. A. Pople, M. Head-Gordon, D. J. Fox, K. Raghavachari, and A. Curtiss, J. Chem. Phys. 90, 5622 (1989).

${ }^{19}$ D. E. Woon and T. H. Dunning, J. Chem. Phys. 98, 1358 (1993).

${ }^{20}$ D. P. O’Neill and P. M. W. Gill, Mol. Phys. 103, 763 (2005). 